\title{
Dirección estratégica urbana, city marketing, gestión de calidad total y desarrollo económico local. Enfoque metodológico
}

\author{
Direção Estratégica Urbana, City Marketing, Gestão da Qualidade Total e \\ desenvolvimento econômico local. Enfoque metodológico \\ Urban Strategic Management, City Marketing, Total Quality Management \\ and local economic development. Methodological approach
}

Amaia Bañales-Mallo[a] [iD, Maria Soledad Aguirre-García[a], María Leticia Santos-Vijande[b] [D]

[a] Universidad del País Vasco/Euskal Herriko Unibertsitatea (UPV/EHU), Facultad de Economía y Empresa, Departamento de Economía Financiera II, Bilbao, España

[b] Universidad de Oviedo (UNIOVI), Facultad de Economía y Empresa, Departamento de Administración de Empresas, Oviedo, España

Cómo citar: Bañales-Mallo, A., Aguirre-García, M. S., \& Santos-Vijande, M. L. (2019). Dirección estratégica urbana, city marketing, gestión de calidad total y desarrollo económico local. Enfoque metodológico. urbe. Revista Brasileira de Gestão Urbana, v.11, e20180205. https://doi.org/10.1590/2175-3369.011.e20180205

\section{Resumen}

Las ciudades tienen un rol cada vez más importante como generadoras de desarrollo económico, son realidades complejas y dinámicas que rivalizan entre sí para atraer recursos, fondos y talento. Así, resulta imprescindible que sean capaces de gestionar con éxito los múltiples desafíos que condicionan su competitividad, haciendo que la gestión urbana constituya un campo de estudio de máxima trascendencia. Este trabajo reivindica el estudio de casos como metodología de gran validez científica para conocer y comprender cómo desarrollar la Dirección Estratégica Urbana (DEU) y sus implicaciones en la competitividad urbana analizada desde una perspectiva económica. Primero, se destaca el carácter científico del estudio de casos como metodología cualitativa. Posteriormente, se propone el diseño metodológico a seguir para garantizar la validez y fiabilidad de los resultados. Finalmente, se ilustra la aplicación de esta metodología en la gestión urbana de un estudio de casos concreto. Los resultados obtenidos muestran que los gestores urbanos parecen priorizan el desarrollo del City Marketing y/o de la Gestión de Calidad Total, y que estas prácticas favorecen, en última instancia, el desarrollo de la DEU y la mejora de la competitividad urbana de modo directo e indirecto.

Palabras clave: Dirección estratégica urbana. Competitividad urbana. Estudio de casos.

\section{Resumo}

As cidades têm um papel cada vez mais importante como geradoras de desenvolvimento econômico, são realidades complexas e dinâmicas que disputam umas com as outras para atrair recursos, fundos e talento. Assim, é imprescindível serem capazes de gerir com sucesso os múltiplos desafios a condicionar sua competitividade, fazendo com que a gestão urbana constitua uma matéria de estudo da maior transcendência.

ABM es doctora en Administración y Dirección de Empresas, e-mail: amaia.banales@ehu.eus

MSAG es doctora en Ciencias Económicas y Empresariales, e-mail: marisol.aguirre@ehu.eus

MLSV es catedrática de Comercialización e Investigación de Mercados, doctora en Ciencias Económicas y Empresariales, e-mail: lsantos@uniovi.es 
Este trabalho reivindica o estudo de casos como metodologia de grande validade científica para conhecer e compreender o como a Direção Estratégica Urbana (DEU) foi desenvolvida e as suas implicações na competitividade urbana analisada numa perspectiva econômica. Antes de tudo, destaca-se o carácter definitivo do estudo de caso como metodologia qualitativa. Depois, propõe-se o projeto metodológico a seguir nos estudos de casos para garantir a validade e fiabilidade dos resultados. Por fim, ilustra-se a aplicação desta metodologia na gestão urbana num estudo de caso particular. Os resultados obtidos mostram como os gestores urbanos parecem priorizar o desenvolvimento do City Marketing e/ou da Gestão de Qualidade Total, e como estas práticas favorecem, afinal, o desenvolvimento da DEU e a melhoria da competitividade urbana seja de modo direto ou indireto.

Palavras-chave: Direção estratégica urbana. Competitividade urbana. Estudo de casos.

\section{Abstract}

Cities have an increasingly important role as generators of economic development. They are dynamic and complex realities that compete with each other to attract resources, funds and talent. Thus, cities need to be able to manage successfully the multiple challenges that condition their competitiveness. Therefore, urban management constitutes a field of study of maximum transcendence. This article claims case study as a methodology of great scientific validity, useful to learn and understand how to implement Urban Strategic Management and its implications in urban competitiveness analyzed from an economic perspective. Firstly, the paper highlights the scientific character of case study as a qualitative methodology. Subsequently, we propose a methodological design to follow in case studies in order to guarantee the validity and reliability of the results. The later section details the execution of this methodology in a specific investigation related to urban management. The results show that urban managers seem to prioritize the development of City Marketing and/or Total Quality Management, and that these practices favor, ultimately, the development of Urban Strategic Management and the improvement of urban competitiveness directly and indirectly.

Keywords: Urban strategic management. Urban competitiveness. Case study.

\section{Introducción}

El objeto de análisis de las Ciencias Sociales es una realidad compleja y cambiante que presenta múltiples interacciones con otros fenómenos circundantes (Ritchie et al., 2014), lo que requiere un estudio pormenorizado de cada situación concreta para poder comprenderla y explicarla adecuadamente. En estos casos, la recopilación y análisis estadístico de datos cuantitativos (metodologías cuantitativas) puede no ser factible y/o suficiente para analizar la realidad objeto de estudio, mientras que las metodologías cualitativas investigan en profundidad el origen, la naturaleza y la evolución de los fenómenos sociales objeto de estudio (Ritchie et al., 2014).

Desde un enfoque riguroso, y muchas veces interdisciplinar, las metodologías cualitativas recurren al estudio de lo particular y generan gran riqueza de detalles que ayudan a conseguir una comprensión integral, real y detallada del fenómeno estudiado (Ritchie et al., 2014), aspectos especialmente útiles en las Ciencias Sociales y, consecuentemente, en la investigación relacionada con la gestión de entidades públicas y privadas (De Massis \& Kotlar, 2014). Así, los métodos cualitativos permiten contribuir positivamente a la eficiencia en la toma de decisiones y al logro de objetivos de cualquier organización.

La metodología cualitativa adquiere especial relevancia en investigaciones descriptivas y exploratorias, muy habituales en el ámbito de las organizaciones (Denzin \& Lincoln, 2005), y prevalece frente a otras alternativas cuando (Ritchie et al., 2014): (1) se pretende comprender el cómo y/o el porqué del fenómeno investigado; (2) el objeto de estudio es multidimensional y complejo y (3) las evidencias e investigaciones de dicho fenómeno son escasas, parciales o limitadas.

El estudio de casos es una de las técnicas más recurrentes en investigaciones cualitativas (Hyett et al., 2014). Consiste en el estudio y análisis sistemático de uno o varios casos en su contexto real, intentando capturar la complejidad del fenómeno objeto de estudio (Yin, 2014). Esta técnica brinda la oportunidad de 
obtener, con gran nivel de flexibilidad, una visión holística y profunda del fenómeno investigado y facilita la comprensión del mismo con un gran nivel de realismo (Hyett et al., 2014).

Sin embargo, la literatura sigue debatiendo la validez y el uso del estudio de casos en la práctica (Hyett et al., 2014), lo que sugiere que la defensa de su rigor y validez como técnica de investigación requiere de una mayor revisión y profundización.

El presente trabajo tiene como propósito en primer lugar, destacar el carácter científico del estudio de casos como metodología cualitativa. En segundo, proponer el diseño metodológico a seguir en los estudios de casos para garantizar la validez y fiabilidad de los resultados obtenidos. En tercer lugar, ilustrar cómo debería aplicarse esta metodología en el desarrollo de un estudio de casos concreto. Se pretende reivindicar el estudio de casos como metodología cualitativa de gran validez científica para conocer y comprender cómo poner en marcha la Dirección Estratégica Urbana (DEU) y sus implicaciones en la competitividad urbana desde una perspectiva económica. La competitividad urbana puede relacionarse con el desarrollo social, económico, cultural, ambiental o político-institucional, entre otros (Fernandes et al., 2018; Palonen, 2011; Weiss et al., 2015). Sin embargo, y reconociendo la existencia de vínculos entre ellos (Fernandes et al., 2018), este artículo se centra principalmente en la competitividad y el desarrollo económico local.

La gestión urbana constituye un campo de estudio de máxima actualidad debido a la importancia creciente de las ciudades y a la dificultad del análisis de cómo afrontar su gobierno de manera eficiente y competitiva (Ye \& Björner, 2018). Las ciudades han adquirido un rol cada vez más importante como generadoras de desarrollo económico que rivalizan entre sí para atraer recursos, fondos y talento, en un intento de mejorar sus resultados (Weiss et al., 2015). Además, la globalización ha incrementado la rivalidad entre ciudades haciendo que el campo de juego sea más amplio, complejo y dinámico (Boisen et al., 2018). En este contexto, las ciudades deben ser capaces de gestionar con éxito los desafíos a los que se enfrentan, consiguiendo ofrecer una propuesta de valor apreciada por los grupos de interés urbanos (residentes, visitantes e inversores) cuya respuesta condiciona, en última instancia, la competitividad urbana (Rasoolimanesh et al., 2014). Así, en la literatura surge el concepto DEU como una prioridad en la gestión para alcanzar una mayor competitividad en las ciudades (Karyotakis \& Moustakis, 2014).

En este entorno, es necesario explorar cómo facilitar en la práctica el desarrollo de la DEU y cómo se relaciona la DEU con la mejora de resultados urbanos. La literatura sugiere que la gestión urbana desde la perspectiva de satisfacción del cliente (denominada City Marketing) o de acuerdo con los principios de Calidad Total, pueden constituir impulsores de la DEU (Wang et al., 2012) y de la mejora de los resultados. No obstante, los estudios publicados hasta ahora sobre la adopción por los gobiernos locales de estos enfoques (City Marketing -CM- o Gestión de Calidad Total -GCT-), y su relación con la DEU y los resultados, han sido escasos y/o parciales (Bañales, 2014). En definitiva, este es un campo de estudio complejo y dinámico, en el que es necesario desentrañar el cómo y el porqué de las relaciones entre las variables consideradas, constituyendo un ámbito idóneo para la metodología cualitativa.

Además, la competitividad urbana y la gestión de los retos que la condicionan son un fenómeno real y actual sobre el que no se tiene control; los límites entre dicho fenómeno y el contexto en el que se da no son precisos; y se busca obtener una visión completa y profunda del mismo capturando su complejidad (Hyett et al., 2014), aspectos que nos hicieron decidirnos por esta metodología.

\section{La investigación cualitativa en la gestión de entidades públicas y privadas y su carácter científico}

El estudio de casos es una metodología empírica cualitativa que consiste en recabar información relevante de casos concretos y analizarla de forma individual, grupal y comparativa (en el caso de recurrir a varios casos), obteniendo unas conclusiones que ayuden a entender holísticamente el fenómeno objeto de estudio en su contexto real (Hyett et al., 2014). Es de las técnicas más habituales en la investigación cualitativa (De Massis \& Kotlar, 2014), y muchos tratadistas la consideran como una de las más útiles en el ámbito de la gestión de las entidades públicas o privadas por el grado de realismo que aporta en sus conclusiones y por la complejidad que comporta comprender la lógica y las implicaciones del comportamiento organizativo (Hyett et al., 2014). Cabe señalar que el estudio de casos maneja información cualitativa y cuantitativa, aunque el aspecto cualitativo suele predominar (De Massis \& Kotlar, 2014).

Pese a su utilidad, el estudio de casos ha sido el centro de varias críticas: 
Sesgo del investigador. El investigador especifica el fenómeno a estudiar, elige el marco teórico, realiza la recogida de datos, pondera la relevancia de las fuentes, las analiza, etc. (Poortman \& Schildkamp, 2012). Esta subjetividad puede conllevar falta de rigor en la recogida, construcción y análisis e interpretación de la evidencia, a lo que podría sumarse la dificultad de tener que manejar la gran cantidad de información que se genera (De Massis \& Kotlar, 2014).

Dificultad de generalizar los resultados. La generalización requiere extrapolación, y en la metodología cualitativa esta no puede ser plenamente justificada porque las muestras utilizadas no tienden a ser elegidas por su representatividad (Poortman \& Schildkamp, 2012); y porque los resultados obtenidos están siempre integrados en el contexto de una situación específica (De Massis \& Kotlar, 2014).

Fiabilidad de los resultados. El investigador no suele poner a disposición de la comunidad científica toda la información que ha utilizado, dificultando que otros puedan llegar a las mismas conclusiones o al menos, entender cómo se ha llegado a estas (Hyett et al., 2014).

Actualmente está aceptado que estas críticas pueden ser superadas si en el desarrollo del estudio de casos se demuestra calidad y rigor, aspectos que la comunidad científica evalúa con los criterios de fiabilidad y validez (De Massis \& Kotlar, 2014).

Fiabilidad: posibilidad de replicar estudios, que diversos investigadores empleen los mismos métodos de recolección de datos y obtengan resultados similares (Riege, 2003). Para ello, se recurre al registro detallado y sistemático del proceso de recogida, análisis e interpretación de los datos (Yin, 2014). Este mecanismo permite replicar la investigación y evaluar su objetividad. La fiabilidad incrementa la confianza en el estudio y evita el sesgo del investigador, venciendo la primera crítica comentada (Hyett et al., 2014).

Validez: valor explicativo de la teoría/resultado que surge de la investigación.

a) Validez de constructo: los indicadores y su definición operativa miden adecuadamente el concepto de estudio (Denzin \& Lincoln, 2005). Para ello se recomienda el uso de múltiples fuentes y métodos de recopilación de evidencia, obteniendo una imagen más precisa de la realidad (Yin, 2014) y limitando la subjetividad (De Massis \& Kotlar, 2014).

b) Validez interna: se establecen correctas relaciones causa-efecto obtenidas de la investigación, tras comprender las razones por las que la relación existe (De Massis \& Kotlar, 2014). Requiere que el patrón de comportamiento se describa de manera plausible y se descarten posibles interpretaciones alternativas (Poortman \& Schildkamp, 2012). En la práctica deben buscarse similitudes y diferencias entre las experiencias estudiadas, aportar argumentos, información suplementaria, comparaciones, comprobaciones, etcétera, demostrando que se ha examinado toda la evidencia relevante, que el análisis se ha enfocado a las preguntas del estudio y que la teoría resultante es consistente con la observación empírica (Villarreal \& Landeta, 2010). Este proceso también restringe subjetividades.

c) Validez externa: grado de generalización de los resultados. Varios autores defienden la generalización analítica ("transferibilidad"-Maxwell, 1998) de los resultados (De Massis \& Kotlar, 2014). Esta generalización puede darse cuando (1) las condiciones teóricas bajo las que se extrajeron los resultados sean similares a las de la nueva situación y (2) se dé la lógica de la réplica (Yin, 2014), es decir, similitudes a partir de dos o más casos similares (replicación literal) y resultados opuestos a partir de dos o más casos en condiciones teóricas diferentes (replicación teórica). Así, resulta importante incluir en el estudio abundante información sobre las condiciones teóricas (personas, organizaciones, contexto, etc.) en las que se ha realizado el estudio (Poortman \& Schildkamp, 2012).

Consecuentemente, existen directrices que ayudan a que las investigaciones cualitativas sean fiables y válidas, haciendo que su carácter científico y su uso esté admitido en muchos ámbitos de estudio, incluido la gestión de las entidades públicas y privadas (De Massis \& Kotlar, 2014).

\section{Diseño metodológico propuesto}

En la literatura de estudio de casos, para asegurar su carácter científico y los criterios de fiabilidad y validez, se han desarrollado varias propuestas metodológicas (Castro, 2010; Chiva, 2001; Eisenhardt, 1989; Villarreal \& Landeta, 2010; Yin, 2014). Aunque todas presentan similitudes importantes, no existe un modelo 
generalmente aceptado que guíe en las labores de recopilación, análisis e interpretación de la información (Bañales, 2014).

Basándonos en dichas propuestas, y con intención de desarrollar un estudio coherente en el que prevalezcan el rigor y la calidad, establecimos un diseño metodológico de estudio de casos (Figura 1). El seguimiento exhaustivo de este protocolo permite realizar un estudio empírico de carácter científico en el que prevalecen fiabilidad y validez. Además, el hecho de que exista un diseño metodológico le aporta fiabilidad al estudio.

El siguiente apartado detalla las fases de este protocolo para una investigación concreta.

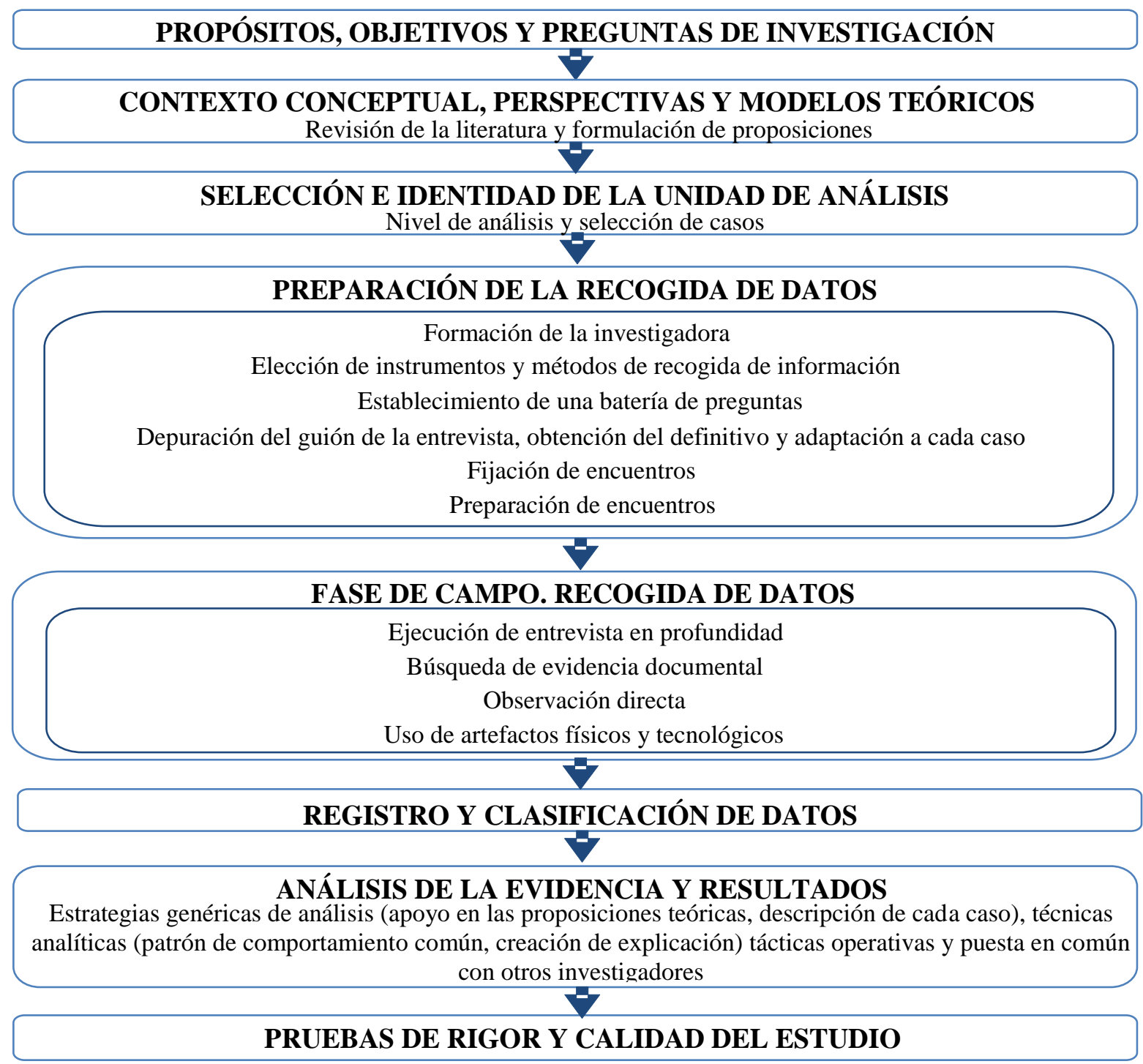

Figura 1 - Diseño metodológico de estudio de casos. Fuente: Elaboración propia (2018).

\section{La investigación}

Propósito, objetivos y preguntas de investigación

Con intención de profundizar en la comprensión de cómo impulsar la competitividad urbana en términos económicos, se fija como objetivo analizar la posible relación existente entre la DEU y los resultados obtenidos en las ciudades desde una perspectiva económica. 
El segundo objetivo establece estudiar el papel que juega la adopción por los organismos gestores de la ciudad de CM y de GCT en el desarrollo de DEU. Ambos enfoques de gestión han sido identificados como útiles para ayudar a avanzar hacia una gestión a largo plazo que contemple, de manera holística, los diferentes ámbitos de gestión urbana y los distintos públicos relevantes a los que aportar valor añadido (Boisen et al., 2018; Karyotakis \& Moustakis, 2014; Pimentel \& Major, 2016; Wiśniewska \& Szczepańska, 2014). También se puede investigar la posible relación de estos enfoques de gestión con los resultados urbanos en términos económicos, de forma directa e indirecta, mediante el apoyo a la DEU. Se busca comprender y explicar relaciones complejas no directamente observables, además de extraer conclusiones prácticas que puedan ser útiles para la gestión de las ciudades.

La gestión de las ciudades es el resultado de la labor y el esfuerzo conjunto y coordinado del sector público, del sector privado y de la sociedad civil, si bien es cierto que es el estamento público el que adquiere el rol promotor principal (Fernandes et al., 2018). El conocimiento de los retos urbanos y la proximidad a los actores urbanos, entre otros, justifican que los ayuntamientos y el equipo de gobierno sean los actores más adecuados para promover y liderar esta gestión (Pascual, 2002). El gobierno de la ciudad además de realizar funciones como institución no lucrativa (administrador de caudales públicos al servicio de la comunidad); como institución política (programas y candidatos); como promotor de cambios sociales (causas sociales) y como proveedor de servicios (servicios públicos), también ejerce de promotor del desarrollo de la propia ciudad (Cervera, 2001).

Consecuentemente, este estudio pretende dar respuesta a las siguientes preguntas de investigación:

1.- ¿Cómo influye la adopción de la DEU por parte de los organismos gestores urbanos en los resultados de la ciudad en términos económicos?

2.- ¿En qué medida el desarrollo del CM contribuye a la adopción de la DEU?

3.- ¿En qué medida el desarrollo de la GCT contribuye a la adopción de la DEU?

4.- ¿Cómo repercute una mayor adopción del CM por parte de los organismos gestores en los resultados de la ciudad en términos económicos?

5.- ¿Cómo repercute una mayor adopción de la GCT por parte de los organismos gestores en los resultados de la ciudad en términos económicos?

\section{Contexto conceptual, perspectivas y modelos teóricos}

El estudio de la literatura relacionada con DEU, CM y GCT nos permite obtener un cuerpo de conocimientos teóricos lo suficientemente importante para contextualizar la investigación, reforzar su relevancia y plantear de forma razonada el modelo teórico a contrastar.

La DEU conlleva un proceso sistemático, creativo y participativo que sienta las bases de una actuación integrada e interdisciplinar a largo plazo. Para su desarrollo, los gobiernos locales necesitan identificar y evaluar de forma continua las oportunidades y amenazas a las que se enfrentan, y sus fortalezas y debilidades con respecto a ciudades rivales. Después, definen el modelo de desarrollo urbano deseado y formulan estrategias y líneas de acción que favorezcan alcanzar dicho modelo, así como los indicadores de seguimiento correspondientes (Johnsen, 2016). Así, la DEU es un proceso continuo de reflexión y de acción que integra la formulación e implementación de las decisiones que permiten al gobierno local alcanzar sus objetivos aportando valor añadido a sus públicos relevantes (Jonhsen, 2016). Conceptualmente, un desarrollo adecuado de la DEU contribuye a que la gestión de la ciudad se haga con visión y eficiencia, obteniendo, por medio del valor añadido entregado, la satisfacción de todos los grupos de interés de la localidad en el largo plazo (Boyne \& Walker, 2010) y, consecuentemente, hace que las localidades sean más atractivas para residentes, inversores y visitantes. Así, la DEU favorece conseguir y mantener una ventaja competitiva sostenible en el tiempo, que haga que la ciudad crezca en competitividad y mejore su posición en el mercado urbano (Rasoolimanesh et al., 2014).

El CM conlleva un enfoque de gestión urbana basado en la necesidad de aportar valor a los visitantes, residentes e inversores, y la operativización de este enfoque mediante el contacto permanente con estos públicos para reconocer sus necesidades y gestionar y comunicar una oferta de ciudad que les aporte valor añadido a largo plazo (Boisen et al., 2018). 
La GCT implica la adopción por los gobiernos locales de un sistema de gestión global que construye una personalidad corporativa única y dinámica, basada en el compromiso de los empleados hacia la entrega de un valor constante a todos los grupos de interés, mediante la mejora continua del conjunto de procesos organizativos (Psomas et al., 2017).

Teniendo esto en consideración, y en base a una profunda revisión de la literatura, se elaboran nueve proposiciones teóricas a analizar y validar que generan un modelo conceptual (Figura 2).

La correcta adopción y puesta en práctica del CM requiere el desarrollo de tres dimensiones:

(1) Comprensión e interiorización del concepto de CM desde una perspectiva amplia, como enfoque de gestión.

(2) Establecimiento de mecanismos que faciliten su operativización, mediante la generación y la diseminación de información y la respuesta mediante el diseño una oferta de valor. Estas actuaciones se denominan orientación al mercado (Kohli \& Jaworski, 1990).

(3) Creación de una imagen en la mente de los públicos objetivo asociada a la marca ciudad que influya positivamente en la diferenciación de la ciudad (city branding).

Conceptualmente, se establece que el CM facilita el desarrollo de la DEU y refuerza la competitividad y los resultados de las ciudades de forma directa y como apoyo al desarrollo de la DEU (Wang et al., 2012). Por tanto, nuestro modelo teórico defiende que el desarrollo del CM es fundamental para mejorar la competitividad urbana de manera indirecta, mediante el desarrollo de la DEU, y de manera directa, mejorando directamente los resultados urbanos en términos económicos.

La forma más habitual en la literatura de clasificar y describir las actuaciones que conlleva la puesta en práctica de la GCT son los modelos de excelencia (Dahlgaard-Park et al., 2018).

En Europa, como referente para los organismos interesados en aplicar la GCT, destaca el modelo EFQM de Excelencia que identifica la necesidad de adoptar las dimensiones: (1) liderazgo; (2) estrategia; (3) personas; (4) alianzas y recursos y (5) procesos, productos y servicios.

Se establece, conceptualmente, que la GCT en el ámbito urbano contribuye a favorecer la DEU, y fomenta la mayor competitividad urbana y los mejores resultados tanto de forma directa como a través de la DEU (Johnsen, 2016; Karyotakis \& Moustakis, 2014; Wiśniewska \& Szczepańska, 2014). Nuestro modelo teórico defiende, por tanto, que la GCT en el ámbito urbano ejerce un efecto directo e indirecto (mediante el desarrollo de la DEU) en la competitividad urbana en términos económicos. 


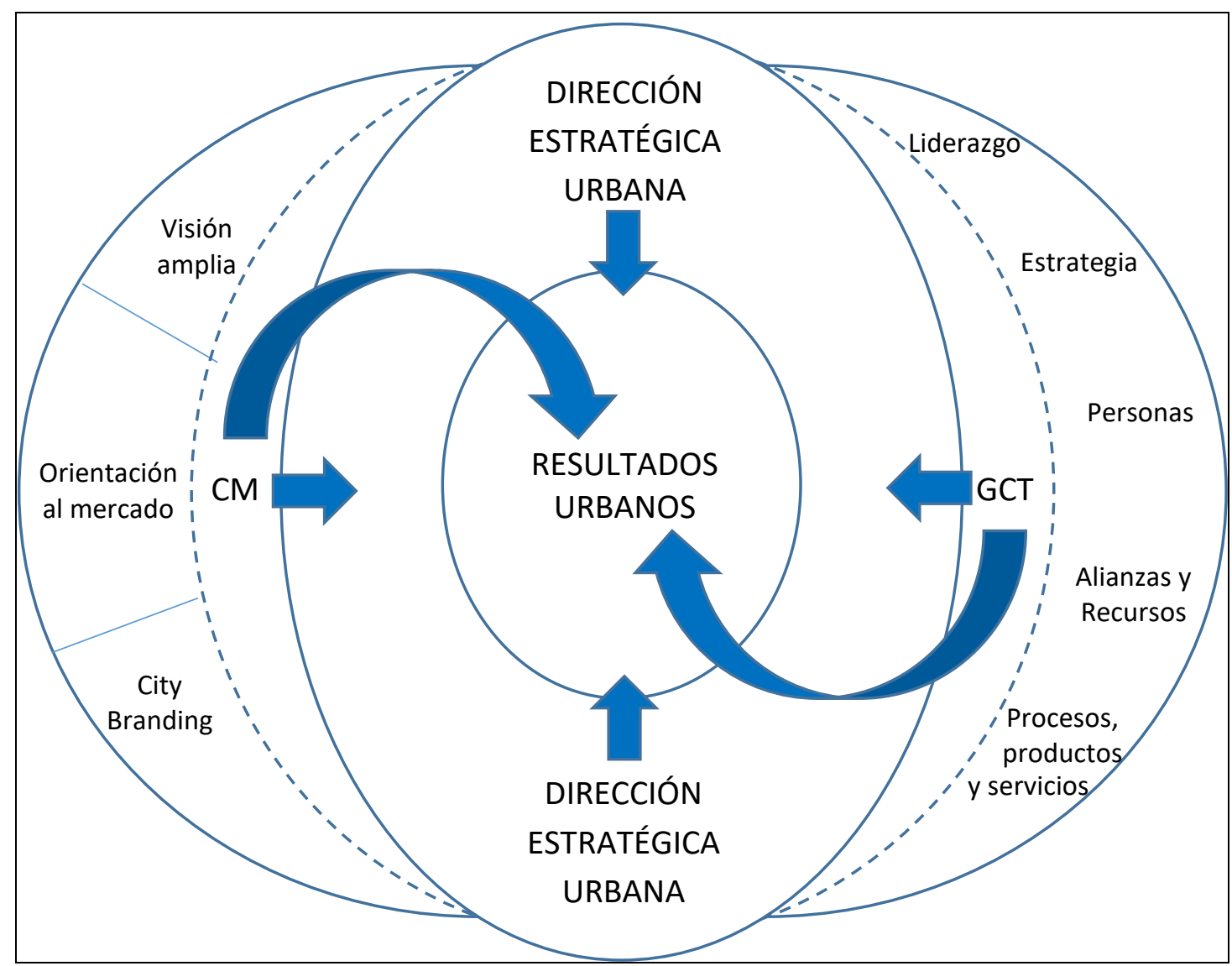

Figura 2 - Modelo conceptual. Fuente: Elaboración propia (2018).

\section{Selección e identidad de la unidad de análisis}

Se eligió un estudio múltiple (frente a un único caso) para aportar validez interna al estudio (Riege, 2003). La probabilidad de buscar similitudes y diferencias entre las experiencias estudiadas, incrementaba la posibilidad de un mejor entendimiento de las relaciones causa-efecto $\mathrm{y}$, como consecuencia, los patrones de comportamiento podrían ser descritos de manera más plausible, evitando subjetividades.

Siguiendo la recomendación de Eisenhardt (1989) de utilizar entre cuatro y diez casos, seleccionamos como unidad de análisis de la gestión urbana la gestión llevada a cabo por los organismos gestores del turismo urbano de cinco ciudades españolas (casos): Oviedo, Gijón, Santander, Bilbao y San Sebastián.

El turismo es un sector estratégico que tiene una fuerte repercusión económica en la ciudad actuando como impulsor en el desarrollo de otros aspectos urbanos (Rivera et al., 2016). Por ello, el desarrollo activo del turismo puede considerarse una estrategia válida para la consecución de una ciudad más competitiva para visitantes, inversores y residentes y constituye un exponente de la gestión de la ciudad de máxima importancia. En la gestión del turismo urbano, como en la de la ciudad, el sector público adquiere el rol promotor principal. Estos organismos gestores del turismo funcionan con presupuesto municipal y tienen como responsables a personas dependientes del gobierno local (concejales, puestos de confianza...). Ello nos hace considerar que la gestión del organismo gestor del turismo responde a la filosofía y principios en los que se basa la gestión del gobierno de la ciudad y, por tanto, resulta ser una unidad de análisis que refleja la gestión de la ciudad en su conjunto.

El universo de investigación se limitó a las 25 ciudades más turísticas de España en volumen de viajeros recibidos en hoteles en el periodo 2012-2016 (Exceltur, 2017).

Con intención de obtener una muestra teórica y lógica con capacidad de generalización analítica, se aplicaron varios criterios de selección (Patton, 1990) entre los que destacamos: 
(1) Homogeneidad: ciudades de tamaño medio, pertenecientes a la cornisa cantábrica que comparten clima, paisaje y orografía y que, al no poder ofrecer turismo de sol y playa, tienen un mayor reto para incrementar su competitividad.

(2) Máxima variación: Variedad en comunidades autónomas (Principado de Asturias (Oviedo y Gijón), Cantabria (Santander) y País Vasco (Bilbao y San Sebastián)) y en tipo de organismo gestor del turismo (área dependiente del ayuntamiento en Oviedo y Santander, organismo público municipal en Bilbao, Gijón y San Sebastián (sociedad anónima)).

Preparación de la recogida de datos

Tabla 1 - Protocolo de búsqueda de evidencias e información

\begin{tabular}{|c|c|}
\hline Etapa 1 & $\begin{array}{c}\text { Formación de los investigadores (bibliografía, documentación y entrevistas con personas } \\
\text { expertas). }\end{array}$ \\
\hline Etapa 2 & 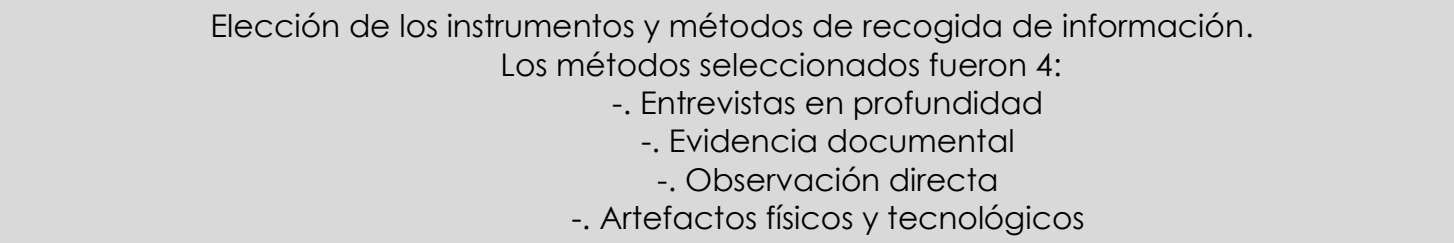 \\
\hline Etapa 3 & Establecimiento de una batería de preguntas (guion de preguntas abiertas) \\
\hline Ełapa 4 & $\begin{array}{l}\text { Depuración del guion de la entrevista, obtención del definitivo (consulta a personas expertas, } \\
\text { prueba piloto) y su correspondiente adaptación a cada caso }\end{array}$ \\
\hline Etapa 5 & Fijación de encuentros \\
\hline Etapa 6 & Preparación de cada entrevista (documentación concreta sobre cada ciudad seleccionada) \\
\hline
\end{tabular}

Fuente: Elaboración propia (2018).

\section{Fase de Campo. Recogida de datos}

Para asegurar la validez de constructo y evitar posibles sesgos, se realizó una triangulación de métodos de recogida de datos y una triangulación de fuentes de información internas y externas, científicas y de divulgación, primarias y secundarias, públicas y privadas, obteniendo información cuantitativa y cualitativa (Denzin \& Lincoln, 2005).

Métodos de recolección de evidencia y fuentes utilizadas:

1) Entrevistas en profundidad. La cualificación y experiencia de los entrevistados proporcionó opiniones de gran valor:

a. Entrevistas previas presenciales, abiertas y no estructuradas con expertos en la materia para profundizar en el conocimiento de la gestión urbana. En un ambiente distendido se pretendía conocer la opinión experta de los entrevistados sobre la utilidad y el grado de aplicación actual por las administraciones públicas de la DEU, el CM, la GCT, la gestión turística de lugares y la gestión urbana en general.

b. Entrevistas en profundidad presenciales, abiertas y semi-estructuradas, con los principales responsables de la promoción turística de las ciudades seleccionadas.

Para asegurar la validez de constructo y confirmar una correcta comprensión, el entrevistador reflejaba lo que iba captando repitiendo ideas o parafraseando (técnica reflejo). Los contactos posteriores también fueron útiles (e-mail y teléfono) para realizar aclaraciones o solicitar documentación adicional.

2) Evidencia documental:

a. Documentación interna: planes estratégicos, de marketing y de turismo de comunidades autónomas y ciudades, autoevaluaciones del modelo EFQM de organismos gestores del turismo, encuestas a ciudadanos, estudios de reputación digital, etc. 
b. Publicaciones de divulgación: notas de prensa, información aparecida en medios de comunicación, libros, páginas web, etc.

c. Publicaciones científicas: artículos, libros y estudios.

d. Estadísticas, informes y bases de datos obtenidas de organismos públicos y privados.

3) Observación directa: siendo turista en las ciudades y acudiendo a las oficinas de turismo en busca de documentación, observamos directamente la situación objeto de estudio.

4) Artefactos físicos y tecnológicos: grabaciones de las entrevistas para su posterior trascripción. Permitió volver sobre la evidencia y confirmar así ciertas afirmaciones o comentarios (Villarreal \& Landeta, 2010).

Así, trazamos una línea de evidencia entre las cuestiones propuestas inicialmente (sujetas a revisión) y las conclusiones finales del estudio (Riege, 2003).

\section{Registro y clasificación de datos}

Se transcribieron las entrevistas y las anotaciones correspondientes a las observaciones directas. La información se archivó en carpetas (digitales y físicas) por cada caso y, posteriormente, se siguió un proceso de estructuración en base a las dimensiones: a) información general de la ciudad, del turismo, su evolución y su organismo gestor en la misma; b) resultados económicos urbanos; c) DEU; d) CM; e) orientación al mercado; f) city branding y g) dimensiones de la GCT.

Así, se obtuvo una base de datos que facilitaba su posterior análisis teniendo en cuenta las proposiciones teóricas establecidas.

\section{Análisis de la evidencia y resultados}

Se siguieron dos estrategias genéricas (Yin, 2014): (1) desarrollo de la descripción de cada caso y (2) apoyo en las proposiciones teóricas obtenidas de la revisión de la literatura. Se describió brevemente cada caso, para pasar posteriormente al análisis de los mismos en base a las proposiciones teóricas.

Las técnicas analíticas utilizadas (Riege, 2003) fueron: (a) construcción de explicaciones, que pretendió establecer vínculos causales entre la gestión desarrollada por los organismos gestores del turismo de las ciudades y los resultados urbanos obtenidos en términos económicos y (b) búsqueda de un patrón de comportamiento, que se vio reflejada en la comparación realizada entre los datos empíricos conseguidos y el marco analítico extraído de la literatura plasmado en la Figura 2.

La táctica que adquirió mayor peso en el estudio consistió en identificar dentro de un grupo de casos las similitudes, y entre los distintos grupos las diferencias relacionadas con unas categorías seleccionadas (Eisendhart, 1989). El análisis realizado trajo consigo la redacción de unos resultados analíticamente generalizables, que fueron objeto de varias conversaciones entre investigadores, subsanando así, el posible riesgo de subjetividad.

\section{Pruebas de rigor y calidad del estudio}

El rigor y la calidad del estudio fueron refrendados en multitud de ocasiones (Tabla 2). 
Tabla 2 - Pruebas de evaluación de calidad y rigor

\begin{tabular}{|c|c|c|}
\hline Prueba & Fase de la investigación & Acciones garantistas \\
\hline \multirow[t]{4}{*}{ Fiabilidad } & $\begin{array}{l}\text { Diseño metodológico de la } \\
\text { investigación }\end{array}$ & $\begin{array}{l}\text { Establecimiento de un diseño metodológico y } \\
\text { seguimiento de sus pautas como guía de acción }\end{array}$ \\
\hline & Preparación de la recogida de datos & $\begin{array}{c}\text { Realización de un protocolo de búsqueda de } \\
\text { evidencias }\end{array}$ \\
\hline & Fase de campo & Seguimiento del protocolo \\
\hline & Registro y clasificación de datos & $\begin{array}{c}\text { Elaboración de una base de datos que organice, } \\
\text { integre y sintetice la información obtenida de las } \\
\text { distintas fuentes de información }\end{array}$ \\
\hline \multirow[t]{8}{*}{$\begin{array}{l}\text { Validez de } \\
\text { constructo }\end{array}$} & $\begin{array}{c}\text { Contexto conceptual, perspectivas y } \\
\text { modelos teóricos. Revisión de la } \\
\text { literatura y formulación de } \\
\text { proposiciones }\end{array}$ & $\begin{array}{c}\text { Análisis en profundidad previo del contexto } \\
\text { conceptual y del marco teórico }\end{array}$ \\
\hline & Preparación de la recogida de datos & Prueba piloto de la entrevista \\
\hline & Fase de campo & $\begin{array}{l}\text { Utilización de distintas fuentes de recogida de la } \\
\text { evidencia (triangulación de fuentes) }\end{array}$ \\
\hline & Fase de campo & $\begin{array}{c}\text { Utilización de distintos métodos de recogida de la } \\
\text { evidencia (triangulación metodológica) } \\
\text {-. Entrevistas en profundidad } \\
\text {-. Documentación } \\
\text {-. Observación } \\
\text {-. Artefactos }\end{array}$ \\
\hline & Registro y clasificación de datos & $\begin{array}{c}\text { Proceso casi simultáneo y unificado de recogida y } \\
\text { análisis }\end{array}$ \\
\hline & Registro y clasificación de datos & Establecimiento de una cadena de evidencia \\
\hline & Fase de campo & $\begin{array}{l}\text { Retroalimentación y contacto interactivo con los } \\
\text { informadores: técnica reflejo y contactos posteriores }\end{array}$ \\
\hline & Fase de Campo & Flexibilidad \\
\hline \multirow[t]{2}{*}{$\begin{array}{l}\text { Validez } \\
\text { interna }\end{array}$} & Análisis de la evidencia y resultados & $\begin{array}{c}\text { Patrón de comportamiento común (apoyo en } \\
\text { proposiciones teóricas) }\end{array}$ \\
\hline & Análisis de la evidencia y resultados & $\begin{array}{l}\text { Creación de explicación (comparación sistemática } \\
\text { de la literatura estructurada en el modelo propuesto) }\end{array}$ \\
\hline \multirow[t]{4}{*}{$\begin{array}{l}\text { Validez } \\
\text { externa }\end{array}$} & $\begin{array}{l}\text { Selección e identidad de la unidad de } \\
\text { análisis. Nivel de análisis y selección de } \\
\text { casos }\end{array}$ & $\begin{array}{c}\text { Establecimiento de unidad de análisis y selección de } \\
\text { casos según el potencial de información sobre el } \\
\text { fenómeno estudiado }\end{array}$ \\
\hline & Métodos y recursos de investigación & $\begin{array}{c}\text { Selección de métodos de recogida de datos } \\
\text { (triangulación metodológica) y de fuentes de } \\
\text { información (triangulación de fuentes) según el } \\
\text { potencial de conocimiento sobre el fenómeno } \\
\text { estudiado. }\end{array}$ \\
\hline & Análisis global y conclusiones & $\begin{array}{c}\text { Aplicación de la lógica replicante (estudio de casos } \\
\text { múltiples) para la consecución de la generalización } \\
\text { analítica }\end{array}$ \\
\hline & Análisis global y conclusiones & $\begin{array}{c}\text { Consideración de parte de los resultados de la } \\
\text { investigación como hipótesis de partida de estudios } \\
\text { de futuras líneas de investigación }\end{array}$ \\
\hline
\end{tabular}

Fuente: Elaboración propia (2018). 


\section{Conclusiones}

Aunque la utilización de las metodologías cualitativas en general y del estudio de casos en particular es creciente, su aceptación en la comunidad científica es relativamente reducida. Sin embargo, su aplicación en una investigación concreta que cumple con las características necesarias para que el uso del estudio de casos sea oportuno, nos lleva a concluir que es una metodología (1) adecuada para comprender en profundidad la realidad de un fenómeno concreto, complejo y multidimensional ya que aporta información muy rica al respecto y (2) de gran validez científica, siempre que se siga un diseño metodológico apropiado y riguroso.

Este trabajo propone un diseño metodológico específico basado en aportaciones previas y aplicado en un estudio de casos múltiple en el ámbito de la gestión urbana con resultados satisfactorios. En la investigación realizada, el uso estricto y preciso del estudio de casos permitió un conocimiento detallado de la gestión urbana desarrollada por las ciudades analizadas, el análisis de la relación existente entre dicha gestión y los resultados urbanos desde una perspectiva económica, así como el establecimiento de un patrón de comportamiento común. Los resultados obtenidos de la contrastación del modelo conceptual nos han ayudado a dar respuesta a las cinco preguntas de investigación establecidas.

El análisis relativo a la primera pregunta concluye que el grado de aplicación de la DEU por los organismos gestores favorece la consecución de unos buenos resultados económicos urbanos en el ámbito turístico, existiendo una asociación directa entre DEU y resultados. La DEU en su afán por ser integradora y ayudar al incremento de la eficacia y eficiencia a largo plazo del organismo gestor de la ciudad, debe incluir aspectos tanto de formulación como de implementación de la estrategia (Johnsen, 2016). La formulación, etapa en la que se establece la dirección hacia la que debe orientarse la organización, se divide en cuatro dimensiones (Revuelto et al., 2012): (1) misión, visión y valores; (2) objetivos; (3) análisis estratégico y (4) plan estratégico; mientras que la implementación, conlleva las acciones realizadas en el despliegue de la estrategia formulada e incluye la propia implementación y el control (Revuelto et al., 2012). Tras analizar estos aspectos, se concluye que Bilbao, San Sebastián y Gijón son las ciudades mejor posicionadas en cuanto al grado de desarrollo de la DEU. En la formulación las tres ciudades tienen definidos con claridad, revisan periódicamente y recogen en documentos públicos (1) misión, visión y valores; (2) retos y objetivos a largo plazo; (3) análisis estratégico basado en la búsqueda y estudio sistemático de información y (4) plan estratégico plurianual. En Gijón y San Sebastián el primer plan estratégico turístico fue para 2008-2011 y los actuales abarcan el 2016-2020 y 2017-2020, respectivamente. En Bilbao, el inicio de la formulación estratégica fue previo (2004) y actualmente, se trabaja en el plan 2019-2025. En la implementación, las tres ciudades han creado un sistema de equipos de trabajo que operativizan la formulación desplegando procesos y acciones y fomentan la implicación de las personas en su desarrollo. Además, con periodicidad se supervisa y evalúa todo, se rediseña y se incorporan mejoras.

Santander y Oviedo muestran menor desarrollo de DEU. El primer plan estratégico turístico de Santander es el actual (2015-2020), teniendo definidos, pero todavía sin revisar, misión, visión, valores, objetivos, retos, análisis estratégico y el propio plan. También han hecho controles periódicos de las acciones establecidas y desplegadas, con lo que se encuentra en un estado inicial-medio.

En Oviedo no hay plan estratégico turístico, pero se admite que una DEU adecuada ayudaría a afrontar los desafíos que presenta la situación del mercado turístico actual. Por ello, consideramos que está en un estado inicial.

La clasificación de las ciudades en base a sus resultados turísticos, medidos en términos de crecimiento del número de viajeros, pernoctaciones, estancia media y grado de ocupación hotelera, resulta ser afín a la enumeración derivada del desarrollo de DEU demostrando la existencia de una vinculación entre ambas.

Igualmente, se corrobora que en las ciudades en las que se demuestra mayor interés por el CM como enfoque de gestión se alcanzan en mayor medida las tres dimensiones establecidas conceptualmente y cuya aplicación práctica queríamos confirmar: (1) comprensión y asunción amplia de este concepto en el plano filosófico o cultural de gestión del organismo urbano; (2) orientación al mercado en términos de generación y diseminación de información y respuesta acorde con las expectativas del mercado urbano; y (3) city branding. Además, se establece que son Bilbao, San Sebastián y Gijón las ciudades mejor posicionadas en el desarrollo del CM. Esta clasificación es análoga a la establecida en base al desarrollo de la DEU, confirmando la relación existente entre ambos conceptos y dando respuesta a la segunda pregunta de investigación. Tal y como se planteaba conceptualmente, la DEU y el CM están basados en la adopción de una perspectiva a largo 
plazo para alcanzar los objetivos fijados por las entidades y generar resultados competitivos (Boisen et al., 2018). Este estudio nos permite concluir, también, que en las ciudades analizadas el CM constituye un antecedente temporal del desarrollo de la DEU y que, consecuentemente, en aquellas ciudades en las que la adopción del CM ha sido más intensa, el desarrollo de la DEU se ha visto favorecido.

Se demuestra también, que la aplicación de la GCT en los organismos gestores del turismo en las ciudades analizadas se sustenta en los cinco agentes facilitadores del modelo EFQM. No obstante, debido al carácter público de los organismos analizados, la gestión de estas dimensiones presenta mayores dificultades de aplicación. La operativización de la dimensión personas, por ejemplo, está limitada por las características propias del colectivo público (alta estabilidad laboral, sensibilidad a la antigüedad y dificultad para recompensar, modificar cargos e influir en las contrataciones) y la incertidumbre política, unida a que los principales responsables de los organismos públicos sean cargos eminentemente políticos, dificulta el compromiso a largo plazo necesario para la correcta adopción del modelo (Stringham, 2004). Sin embargo, son dos dificultades que pueden gestionarse y superarse. En este proceso, han sido San Sebastián, Gijón y Bilbao las que han demostrado un mayor desarrollo del modelo EFQM, quedando Santander y Oviedo en un estadio inicial-medio. Podemos, por tanto, establecer que la distribución de las ciudades en base a su adopción de la GCT y de la DEU es muy similar, demostrando la vinculación entre ambas y respondiendo a la tercera pregunta de investigación. Así, como se muestra conceptualmente, la DEU y la GCT están basadas en una profunda reflexión estratégica en las organizaciones y en la toma de decisiones coherentes que buscan la satisfacción de los diversos grupos de interés de manera continua (Psomas et al., 2017), y la generación de resultados competitivos. En los casos estudiados, se demuestra que la adopción del modelo EFQM ha generado mecanismos, conocimiento y experiencia suficientes como para sustentar el desarrollo de la DEU. Esta investigación confirma, además, que el desarrollo de la GCT por los organismos gestores de turismo en las ciudades estudiadas ha sido anterior a la definición de un proceso de DEU.

Indagando en la cuarta pregunta de investigación se establece que el mayor desarrollo del CM por los organismos gestores de la ciudad contribuye a la mejora de los resultados urbanos desde una perspectiva económica, existiendo una asociación directa entre ambos que queda manifiesta en el orden de las ciudades analizadas en cuanto al desarrollo de los dos ítems. La adopción del CM conlleva crear, comunicar, entregar e intercambiar una oferta urbana que satisfaga las necesidades, los deseos y las expectativas de los stakeholders relevantes en el largo plazo (Boisen et al., 2018). Para ello, las ciudades analizadas utilizan (aunque con diferentes grados de desarrollo) mecanismos como: analizan la situación, establecen estrategias de segmentación y posicionamiento, trabajan aspectos tanto tangibles como intangibles del producto urbano, comunican la imagen de la ciudad, diseminan y dan respuesta a la información generada, etc. y todo ello con una clara visión de aportar valor a los clientes en el largo plazo. Así, se consigue incrementar el atractivo de las ciudades, se obtienen ventajas competitivas sostenibles en el tiempo y, consecuentemente, una mejora de los resultados urbanos (Boisen et al., 2018).

También se concluye, dando respuesta a la quinta pregunta de investigación, que el mayor grado de desarrollo de la GCT en el ámbito urbano se asocia positivamente con mejores resultados turísticos en términos económicos de todas las ciudades. Así, por ejemplo, las personas que ejercen el liderazgo en las ciudades analizadas buscan la mejora del sistema de gestión y del rendimiento, aunque el despliegue de sus funciones en este proceso no se realice del mismo modo. En todos los casos se concede gran importancia a la determinación de la estrategia, aunque no en todas las ciudades está definida y operativizada con un mismo grado de desarrollo. El número de mecanismos formales, estructurados y supervisados para motivar e implicar al personal y para estructurar las alianzas y los recursos varía entre ciudades. Además, y aunque el grado de definición de procesos no sea igual, en todas las ciudades diseñan y gestionan sus productos y servicios buscando la optimización de las experiencias para sus grupos de interés. De hecho, la adopción de la GCT genera ciertas rutinas y pautas de actuación en la organización (Dahlgaard-Park et al., 2018), y el mayor grado de la definición y estructuración de estas pautas conlleva un incremento de la eficiencia organizativa de forma constante y permite la obtención de mejores resultados a largo plazo (Psomas et al., 2017).

Se concluye que los gestores urbanos, preocupados por el reconocimiento de sus actuaciones, parecen priorizar el desarrollo de CM y/o de GCT, y que estas prácticas favorecen, en última instancia, el desarrollo de DEU y la mejora de la competitividad urbana desde una perspectiva económica de modo directo e indirecto. 
Otra evidencia obtenida es que la descentralización en el gobierno local y la contratación de gestores profesionales, parecen favorecer un mayor desarrollo del CM y la GCT, reforzando consecuentemente la DEU y los mejores resultados urbanos. Las tres ciudades en las que el turismo no es gestionado directamente por el propio consistorio, y que tienen organismos públicos específicos para su gestión (Bilbao, Gijón y San Sebastián), han resultado ser las ciudades en las que el grado de desarrollo de CM, de GCT y de DEU es mayor.

Además, se demuestra que el grado de desarrollo del CM, la GCT y la DEU en las ciudades de la comunidad autónoma del País Vasco/Euskadi (Bilbao y San Sebastián) es considerablemente elevado. Esto puede estar relacionado con la existencia de (1) la Agencia de Turismo Vasca (BASQUETOUR) que tiene un enfoque completo e integrado y alto nivel y capacidad de planificación demostrada y (2) el Observatorio Turístico de Euskadi, organismo concebido y diseñado para el seguimiento de la actividad turística en el País Vasco y potenciar la gestión inteligente de la información y el conocimiento.

No ocurre lo mismo en el Principado de Asturias, donde el desarrollo de CM, GCT y DEU en Oviedo y Gijón es considerablemente diferente.

Consecuentemente, afirmamos que además de la tipología de organismo gestor, el apoyo supramunicipal a nivel autonómico también facilita el desarrollo de estos enfoques y el incremento de la competitividad urbana.

En definitiva, el estudio llevado a cabo ha permitido establecer un patrón de comportamiento común en la gestión urbana. En el mismo, se establece que aquellas ciudades que tienen un menor desarrollo de CM, GCT y DEU obtienen peores resultados turísticos y nuestro estudio sugiere que una gestión más eficiente de dichas variables permitiría obtener mejores resultados desde una perspectiva económica. Además, la constatación llevada a cabo sobre la existencia de relaciones entre las variables consideradas, conlleva un claro beneficio para los organismos gestores de la ciudad. Así, centrándose en el CM o la GCT y desarrollándolas con eficiencia, se facilitará el futuro desarrollo de la DEU obteniendo desde el inicio mejoras en sus resultados económicos de la ciudad. Todo ello, constituye un avance científico en el ámbito de estudio y resulta de utilidad para los organismos gestores de las ciudades en su toma de decisiones.

Como ocurre en toda investigación, el presente trabajo también tiene ciertas limitaciones a tener en cuenta a la hora de interpretar y hacer uso de las conclusiones obtenidas. Primeramente, el desarrollo local y la competitividad urbana han sido analizados desde una perspectiva fundamentalmente económica, dejando de lado aspectos sociales, ambientales, culturales, etc. Si bien es cierto que coincidimos con Fernandes et al. (2018) al afirmar que existen vínculos entre la economía, la tecnología, la cultura, la creatividad, el bienestar y la calidad de vida de las poblaciones, consideramos que, a la hora de conseguir una visión más holística del desarrollo urbano, resultaría de gran interés ahondar en otros aspectos. Posteriormente, debido a la complejidad y multidimensionalidad de la ciudad y su gestión, en el trabajo realizado nos hemos centrado en el ámbito turístico para analizar la gestión de la ciudad y el grado de competitividad urbana y sería interesante y enriquecedor profundizar en otros ámbitos urbanos. Además, la inexistencia de una base de datos objetivos y oficiales relativos al grado de competitividad urbana nos ha llevado a utilizar los resultados turísticos obtenidos a través de la encuesta hotelera desarrollada por el INE. Estos datos tienen la ventaja de estar publicados por un organismo oficial, de facilitar la comparabilidad entre las ciudades analizadas y de incluir aspectos tanto de la oferta turística hotelera como de la demanda (el aspecto más representativo del turismo estatal), además de representar en gran medida la competitividad urbana. Sin embargo, la competitividad es un fenómeno muy amplio como para captarlo plenamente a través de un solo indicador y es un aspecto en el que se podría ahondar. Otro aspecto relevante y que constituye una limitación de nuestro trabajo tiene que ver con el carácter transversal del turismo. Nuestra unidad de análisis la han formado los organismos responsables del turismo, cuya gestión repercute en gran medida en los resultados turísticos urbanos. No obstante, existen otras muchas variables no dependientes del organismo gestor y, en ocasiones, ni siquiera dependientes del municipio, que pueden influir en los resultados turísticos y que no han sido contempladas en este estudio.

\section{Referencias}

Bañales, A. (2014). Dirección estratégica de las ciudades: impacto del city marketing y del modelo EFQM de excelencia en los resultados urbanos (Tesis doctoral). UPV/EHU, Bilbao, España. 
Boisen, M., Terlouw, K., Groote, P. \& Cowenberg, O. (2018). Reframing place promotion, place marketing, and place branding - moving beyond conceptual confusion. Cities, 80, 4-11.

Boyne, G. A. \& Walker, R.M. (2010). Strategic management and public service performance: the way ahead. Public Administration Review, 70(1), 185-192.

Castro, E. (2010). El estudio de casos como metodología de investigación y su importancia en la dirección y administración de empresas. Revista nacional de administración, 1(2), 31-54.

Cervera, A. (2001). La gestión del marketing en la administración pública local. Dirección y Organización, 26, 113-124.

Chiva, R. (2001). El estudio de casos explicativo. Una reflexión. Revista de Economía y Empresa, 15(41), 119-132.

Dahlgaard-Park, S.M., Reyes, L. \& Chen, C.K. (2018). The evolution and convergence of total quality management and management theories. Total Quality Management and Business Excellence, 20(9-10), 1108-1128.

De Massis, A. \& Kotlar, J. (2014). The case study method in family research: Guidelines for qualitative scholarship. Journal of Family Business Strategy, 5, 15-29

Denzin, N.K. \& Lincoln, Y.S. (2005). The Sage handbook of qualitative research (3a ed.). California: Sage Publications.

Eisenhardt, K.M. (1989). Building theories from case study research. Academy of Management Review, 14(4), 532-550.

Exceltur (2017). UrbanTUR 2016. Monitor de competitividad turística de los destinos urbanos españoles. Madrid: Exceltur.

Fernandes, R., Gama, R., \& Barros, C. (2018). Atividades criativas, especialização inteligente e oportunidades para os territórios urbanos de pequena dimensão: o caso do carnaval de Estarreja. Revista Brasileira de Gestão Urbana, 10 (supl.), 212-227.

Hyett, N., Kenny, A. \& Dickson-Swift V. (2014). Methodology or method? A critical review of qualitative case study reports. International Journal of Qualitative Studies on Health and Well-being, 9(1), 1-12.

Johnsen, A. (2016). Strategic planning and management in local government in Norway: Status after three decades. Scandinavian Political Studies, 39(4), 333-365.

Karyotakis, K. M. \& Moustakis, V.S. (2014). Reinvention of the public sector: Total Quality Management and Change Management. Singidunum Journal of Applied Sciences, 11(2), 30-44.

Kohli, A. K. \& Jaworski, B.J. (1990). Market Orientation: The Construct, Research Propositions, and Managerial Implications. Journal of Marketing, 54(2), 1-18.

Maxwell, J. A. (1998). Designing a qualitative study. En L. Bickman \& D.J. Rog (coord.) Handbook of applied social research methods (pp. 69-100). California: Sage Publications.

Palonen, E. (2011). European Capitals of Culture and the limits of the urban effects in Luxembourg and Sibiu 2007. Revista Brasileira de Gestão Urbana, 3(2), 245-256.

Pascual, J.M. (2002). La gestión estratégica de las ciudades. Un instrumento para gobernar las ciudades en la era infoglobal. Sevilla. Consejería de Gobernación. Junta de Andalucía.

Patton, M.Q. (1990). Qualitative evaluation and research method (2a ed.). California: Sage Publications.

Pimentel, L. \& Major, M. (2016). Key success factors for quality management implementation: evidence from the public sector. Total Quality Management and Business Excellence, 27(9-10), 997-1012.

Poortman, C.L. \& Schildkamp, K. (2012). Alternative quality standards in qualitative research. Quality \& Quantity, 46(6), 1727-1751.

Psomas, E., Vouzas, F., Bouranta, N. \& Tasiou, M. (2017). Effects of total quality management in local authorities. International Journal of Quality and Service Sciences, 9(1), 41-66.

Rasoolimanesh, S.M., Jaafar, M. \& Badarulzaman, N. (2014). Examining the contributing factors for the successful implementation of city development strategy in Qazvin City, Iran. Cities, 41, 10-19.

Revuelto, L. R., Balbastre, F. B. \& Redondo, A. R. (2012). Principios cooperativos y aplicación del modelo EFQM: efectos en el proceso estratégico de la cooperativa de enseñanza "La Nostra Escola Comarcal". CIRIEC-España, 76, $229-259$. 
Riege, A. M. (2003). Validity and reliability tests in case study research: a literature review with "hands-on" applications for each research phase. Qualitative Market Research: An International Journal, 6(2), 75-86.

Ritchie, J., Lewis, J., McNaughton Nicholls, C. \& Ormston, R. (2014). Qualitative research practice. California: Sage Publications.

Rivera, M., Croes, R. \& Lee, S. H. (2016). Tourism development and happiness: A residents' perspective. Journal of Destination Marketing and Management, 5(1), 5-15.

Stringham, S.H. (2004). Does quality management work in the public sector? Public Administration and Management: an Interactive Journal, 9 (3), 182-211.

Villarreal, O. \& Landeta, J. (2010). El estudio de casos como metodología de investigación científica en dirección y economía de la empresa. Una aplicación a la internacionalización. Investigaciones Europeas de Dirección y Economía de la Empresa, 16(3), 31-52.

Wang, C. H., Chen, K. Y. \& Chen, S.C. (2012). Total quality management, market orientation and hotel performance: The moderating effects of external environmental factors. International Journal of Hospitality Management, 31, 119-129.

Weiss, M. C., Bernardes, R.C. \& Consoni, F. L. (2015). Cidades inteligentes como nova prática para o gerenciamento dos serviços e infraestruturas urbanos: a experiência da cidade de Porto Alegre. Revista Brasileira de Gestão Urbana, 7(3), 310-324.

Wiśniewska, M. \& Szczepańska, K.A. (2014). Quality management frameworks implementation in Polish local governments. Total Quality Management, 25(4), 352-366.

Ye, L., \& Björner, E. (2018). Linking city branding to multi-level urban governance in Chinese mega-cities: A case study of Guangzhou. Cities, 80, 29-37.

Yin, R.K. (2014). Case study research. Design and methods (5a ed.). California: Sage Publications.

Editores invitados: Janaina Camile Pasqual Lofhagen, Pontifícia Universidade Católica do Paraná - PUCPR; Christopher Hawkins, University of Central Florida - UCF

Recibido: Nov. 13, 2018

Aprobado: Jul. 24, 2019 